\title{
Form design in interface of display and control system based on visual search
}

\author{
Dayan Wang ${ }^{2}$, Jue $\mathrm{Qu}^{1,2}$, Wei Wang ${ }^{1, *}$, Shuai $\mathrm{Zhu}^{1}$, and Kang $\mathrm{Li}^{1}$ \\ ${ }^{1}$ School of Aeronautics, Northwestern Polytechnical University, Xi'an China \\ ${ }^{2}$ Air and Missile Defense College, Air Force Engineering University, Xi'an China
}

\begin{abstract}
To explore the design pattern and guidelines for the forms displayed on the interfaces, we studied the factors of form design from the perspective of ergonomics. These factors include orientation (horizontal, vertical), color grouping and color combination. In combination with the analysis and conclusion of the experimental data, we proposed some design guidelines for human-computer interface that has many forms, such as weapon command and control system. A visual searching program was designed to simulate the display interface. Reaction time and accuracy are recorded by orthogonal experiment. Analyses such as double factor variance analysis, simple main effect are conducted on the experimental data. The following conclusions are obtained: two-color form has a faster reaction time than single color form; reaction time of the horizontal form is shorter than the vertical form. Color combination has significant influence on accuracy, and the blue-green is suiTable for form design; color grouping has significant influence on reaction time and accuracy; the interaction of color grouping and color combination shows significant differences. When designing forms in display interface, we should choose the horizontal layout with sparse coloring density as much as possible, and the effect of color combination on the recognition efficiency should be considered.
\end{abstract}

\section{Introduction}

The form of war and the characteristics of war under informatization conditions require that combatants must have rapid response and decision-making capabilities [1]. The display and control modes of human-machine interface of aircraft, submarine, ship, anti-aircraft missile and other weapons and equipment display control system feature multi-tasking, multiinformation and multi-mode [2]. If the designs of display quantity, display mode, display timing, size, color, and layout are unreasonable, operators will bear the excessive physiological and psychological endurance, and have more difficulty in information identification, judgment and operation control, which will cause human error during operations [3]. Reference [4] shows that the improvement of human-machine interface can effectively reduce the occurrence of human error, and the effect of human error reduction is significant, especially for unskilled operators. Reference [5] shows that even if the

\footnotetext{
*Corresponding author: 1729581429@.qq.com
} 
appropriate personnel are selected and trained for combat missions through various testing methods, and the training procedures for combatants are improved, the operator cannot safely operate some complicated weapons and equipment. Frequent accidents make people realize that only when weapons and equipment meet the physiological, psychological characteristics and ability limits of users, can the effectiveness of weapons be exploited to avoid accidents. Regarding the ergonomic design of the human-machine interface of weapons and equipment, many research institutions have carried out a lot of research work. Mosier [6] and Sullivan [7] studied the display interface of the aircraft cockpit, analyzed the influence of character, symbol, color, and other information coding methods on the identification performance, and studied the usability level of the interface design and its evaluation method. Yuan Xiuqian, Zhou Qianxiang, Zhuang Damin and others from Beijing university of Aeronautics and Astronautics have focused on the human-machine interface of the aircraft cockpit [8-11]. They analyzed the problems and deficiencies in the ergonomic design of the aircraftcockpit, combined with the mental workload of the pilot, optimized the design and comprehensive evaluation of the display interface of the cockpit. A series of experimental studies have been carried out on the pilot's situational awareness and situational cognition, which provided a scientific basis for the design of information display interface from the perspective of cognition. In the field of Graphical User Interface, a large amount of research has focused on the information display coding of text, icons, meters, etc. [12-17], and few design guidelines have been proposed on the way how forms should be designed, especially in terms of human visual search characteristics.

\section{The characteristics of human visual search}

Human vision search has many characteristics, which are used in interface design. The horizontal movement ability of human eyes is stronger than that of the vertical direction, and the judgment of position, size, color and other attributes is more accurate than that of the vertical direction [18]. Feature integration theory [19] considers that there are two information processing processes in visual search. The first is the pre-attention stage, in which the visual system processes all the stimuli in the field of vision simultaneously. In this stage, attention is not required and only independent features are detected while the relations between the features are not detected. The second is the sequence processing stage with memory capacity limitation. This process connects the independent features of the preattention stage to form a holistic cognition of an object. Bichot et al. [20] proved the existence of the pre-attention phase from the perspective of neurophysiology. Wolfe et al. [21] analyzed the influencing factors and characteristics of the pre-attention phase in detail, and the color was considered to be the most important factor that affects the pre-attention information processing. In the visual search, the subjects will group the forms according to color grouping in the pre-attention stage. Wang Haiyan [22] and others analyzed the visual search behavior of the icons through eye movement experiments and also proved the existence of this grouping strategy.

When designing the forms, the design factors of great significance are orientation (horizontal layout, vertical layout) and color. To this end, this paper takes the orientation, color combination and color grouping of forms as the research objects, and uses Chinese characters and numbers as information display content in forms. The study analyzes the results from the perspective of visual search and provides an ergonomic basis for the designs of forms in display interface. 


\section{Experimental design}

\subsection{Participant}

Overall 34 participants (19 male, 15 female), aged 18 25, participated in this experiment. All the participants are right-handed, healthy, correct vision, no color blindness, weak color and so on. None of the participants tested have been exposed to similar experiments.

\subsection{Experimental equipment}

The experimental equipment includes a HP xw9400 workstation. The experiment is presented on a 22 -inch display screen with a resolution of $1280 \times 1024$ pixels, a screen brightness of $300 \mathrm{~cd} \cdot \mathrm{m}-2$, a bright spot diameter of $0.33 \mathrm{~mm}$, and a refresh rate of $200 \mathrm{~Hz}$. The subjects are about $600 \mathrm{~mm}$ from the screen. The line of sight is at an angle of $90^{\circ}$, and the center of the screen is substantially horizontal to the eye of the test subject.

\subsection{Experimental tasks and interface design}

\section{1) Experiment variables}

Currently, the layout methods commonly used in forms in the weapon equipment display interface are horizontal layout and vertical layout. The commonly used colors are mainly red, green, blue, etc. [23] only studied the effect of orientation on the Easy Search Rate of the forms. [24] selected color and distance as the factors that influence the design of forms. No relevant paper has been reported that takes all the factors into consideration. Based on this, in order to explore the design method of forms in the weapon equipment display interface that is more in line with the human visual search characteristics, a total of three experimental variables are designed for this experiment: two form orientations (horizontal, vertical), six color combinations (red, green, blue, combination of red and green, green and blue, blue and red). In the form filled with combined colors, there are three ways of color groupings $(1 \times 12,3 \times 4,6 \times 2)$ representing different densities. This test, which uses within-subjects design, covered a total of 24 sets of experimental materials.

2) Experimental interface

According to the color attribute of the commonly used forms in the display interface of the weapon equipment display control system, the background of the display panel is set to be black, the characters are white, and the lines are white. Select the commonly used colors: red (RGB; 255, 0, 0), green (RGB; 0, 176, 80), blue (RGB; 0, 112, 192), two of which are combined to get three combined color modes: red green, green blue, blue red. The three monochromatic and three combined colors form a total of six color attributes, and every form contains 24 rectangular areas of the same size, each of which is $1.2 \mathrm{~cm}$ long and 0.6 $\mathrm{cm}$ wide. This format of form is also often used in computer operating systems. Fully simulate the display format of forms in the weapon equipment display interface, divide the experimental forms into two layers: one is composed of Chinese characters, and the other is composed of numbers. In order to reflect the influence of Chinese characters on the display of the Table, the Chinese character layer experiment is composed of four Chinese characters, namely "You", "Shen", "Tian" and "Jia". There are 12 combinations. Taking into account of the symmetry of the numbers on the keyboard, the numbers are combined with " $1 "$, " 2 ", "4", and "5", which correspond one-to-one with Chinese characters, as shown in Fig. 1. 


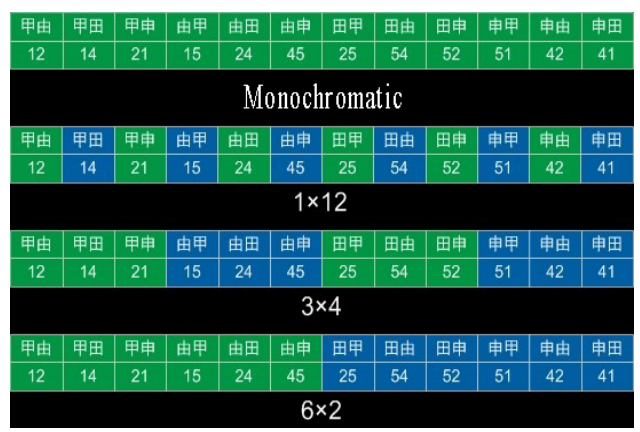

(a) Horizontal layout

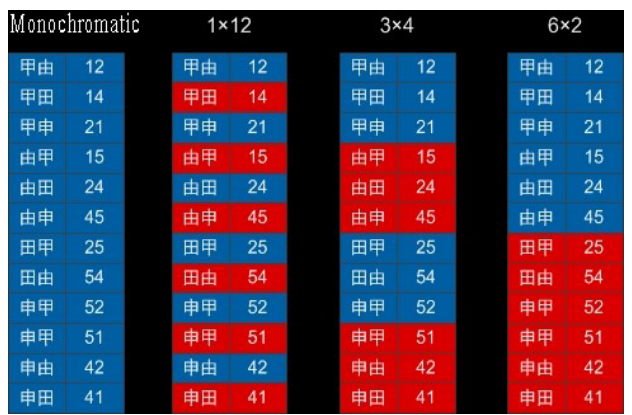

(b) Vertical layout

Fig. 1. Table design for different layout methods and coloring patterns.

3) Experimental tasks

The experimental interface is shown in Fig.2. The participant first searches for the specified Chinese character combination according to the prompt, then searches for the number corresponding to the Chinese character combination, and inputs the number from the keyboard. The experimental procedure is mainly compiled by the standardized psychological experiment system E-Prime software to present the experimental target. The software automatically records the reaction time and accuracy of the participants.

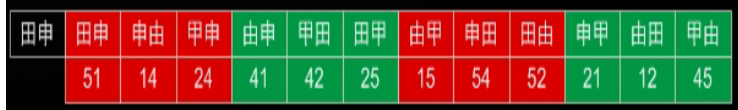

Fig. 2. Experimental interface.

\subsection{Formal experiment}

Step 1: Before the test, the participants are required to take a good rest and maintain a good mental state. The participants are informed of the instructions and allowed to practice 10 times to ensure that the they are fully familiar with the experimental process.

Step 2: Before the formal test, let the participants look at the "+" icon in the center of the screen, make sure that the visual center of the participant is at the center of the screen, and then randomly present a set of test pictures so that the they can be familiar with the test process and ready to start the experiment.

Step 3: The experiment is officially started. Each subject is required to complete 24 different types of form experiments. Each group of experiments has a total of 12 pictures, and each combination of Chinese characters and numbers are randomly distributed. The target area and the target are uniformly presented in the search range, and the participants 
are required to select the prompt target along side the form from the 12 randomly arranged and non-repeated Chinese character combinations of each set of search interfaces under the condition of ensuring the accuracy as much as possible. Enter its corresponding number through the keyboard. After the entering the number, the experiment automatically enters the next interface. In order to ensure that the participants have a good mental state, they can rest according to their subjective wishes in each experimental interval.

Step 4: The tests mentioned above are performed on 34 experimenters until the end of the experiment.

\section{Analysis of the experimental results}

In the experiment, 34 participants participate in 24 different experiments to search for 12 different targets. A total of 9792 data are obtained, of which 384 are incorrectly selected and the error rate is $3.92 \%$. The invalid data are excluded and the analysis of variance is performed on the remaining experimental data.

\subsection{Influence of layout mode, color and colouring mode on reaction time}

The experiment records the results of 34 subjects. As shown in Table 1, the layout method, color combination and color grouping are used as the three factors affecting the reaction time, and the differences between groups are analyzed.

Through the analysis of variance, the orientation $(\mathrm{f}=10.506, \mathrm{p} \leq 0.01)$ and color grouping $(\mathrm{f}=21.376, \mathrm{p} \leq 0.01)$ have significant influence on the reaction time, and the interaction between them $(\mathrm{f}=0.480, \mathrm{p}=0.846)$ is not that obvious. The color is not significant for the reaction time regardless of the monochrome $(\mathrm{f}=0.037, \mathrm{p}=0.776)$ or the two colors $(\mathrm{f}=0.925$, $\mathrm{p}=0.342$ ). Orientation and color combination (monochrome: $\mathrm{f}=0.003, \mathrm{p}=0.863$; two colors: $\mathrm{f}=0.591, \mathrm{p}=0.743)$, color combination and color grouping $(\mathrm{f}=2.304, \mathrm{p}=0.763)$ are not significant for the reaction time.

The average response time of the horizontal layout is $4653 \mathrm{~ms}$, while the average response time of the vertical layout is $5233 \mathrm{~ms}$, and the horizontal layout is $11.08 \%$ faster than the vertical one. In addition, as shown in Fig.3, the thinner the color grouping distribution, the longer the reaction time. The average reaction time of the monochrome is $5436 \mathrm{~ms}$, the average reaction time of the $1 \times 12$ color grouping is $5208 \mathrm{~ms}$, the average reaction time of the $3 \times 4$ is $4771 \mathrm{~ms}$, and the reaction time of the $6 \times 2$ is $4495 \mathrm{~ms}$. There is a significant difference in reaction time between the coloring groupings. The specific values are shown in Table 2.

Table 1. Significant analysis of reaction time by various factors.

\begin{tabular}{c|c|cc}
\hline \hline \multicolumn{2}{c|}{ Factor } & $\mathrm{F}$ & $\mathrm{P}$ \\
\hline \multicolumn{2}{c}{ Orientation } & 10.506 & 0.002 \\
\hline $\begin{array}{c}\text { Color } \\
\text { combination }\end{array}$ & Monochrome & 0.037 & 0.776 \\
\cline { 2 - 4 } & Two-tone & 0.925 & 0.342 \\
\hline \multicolumn{2}{c}{ Color grouping } & 21.376 & 0.004 \\
\hline $\begin{array}{c}\text { Orientation \& } \\
\text { Color } \\
\text { combination }\end{array}$ & Monochrome & 0.003 & 0.863 \\
\cline { 2 - 4 } & Two-tone & 0.591 & 0.743 \\
\hline $\begin{array}{c}\text { Orientation \& Color grouping } \\
\text { Color combination (two colors } \\
\text { only) \& Color grouping }\end{array}$ & 0.480 & 0.846 \\
\hline \hline
\end{tabular}


Table 2. Difference analysis between groups for each coloring mode.

\begin{tabular}{ccc}
\hline \hline Variance analysis & \multicolumn{2}{c}{ Variance analysis } \\
\cline { 2 - 3 } combination & $\mathrm{F}$ & $\mathrm{P}$ \\
\hline Monochrome \& $(1 \times 12)$ & 5.632 & 0.048 \\
Monochrome \& $(3 \times 4)$ & 32.057 & 0.003 \\
Monochrome \& $(6 \times 2)$ & 55.226 & 0.001 \\
$(1 \times 12) \&(3 \times 4)$ & 15.473 & 0.000 \\
$(1 \times 12) \&(6 \times 2)$ & 28.562 & 0.002 \\
$(3 \times 4) \&(6 \times 2)$ & 11.319 & 0.004 \\
\hline \hline
\end{tabular}

Fig 3. shows the reaction time of different color groupings. For a two-color form, the thinner the color grouping distribution, the longer the reaction time, and the linear relationship. In general, the two-color form has a faster reaction time than the monochrome. The average reaction time of the two-color is $4753 \mathrm{~ms}$, which is $9.34 \%$ faster than the monochrome, and the effect is significant $(\mathrm{F}=31.226, \mathrm{P} \leq 0.001)$.

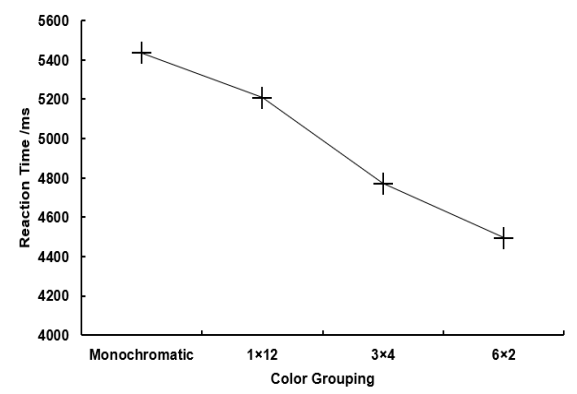

Fig. 3. The reaction time of different color groupings.

According to the previous analysis, the monochrome form has a longer reaction time than the two-color, and the difference in reaction time is remarkable. Therefore, color analysis is divided into two categories, one is monochrome color analysis, and the other is two-color analysis. For the color of the monochrome form, the difference in reaction time is not significant $(\mathrm{f}=0.037, \mathrm{p}=0.776)$, the reaction time of green is the shortest, the reaction time blue is the longest, and the difference between the groups is not significant. For the two-color form, the color combination has no significant effect on the reaction time $(\mathrm{f}=2.340, \mathrm{p}=0.615)$, and the difference between the color combinations is not significant. Draw a line chart based on the specific reaction time of the color, as shown in Fig. 4.

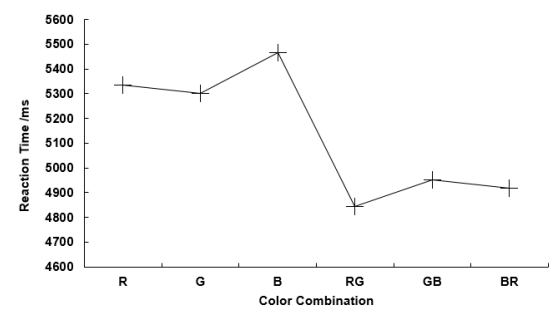

Fig. 4. Average reaction time under each color attribute.

\subsection{Influence of layout mode, colour and colouring mode on the accuracy}

Because there is an intrinsic relationship between reaction speed and accuracy, there is a trade-off between the two. If the reaction time is studied separately and the accuracy is not 
studied, it is difficult to reflect the influence of each factor on the identification performance. However, the error option in the experimental data only accounts for $3.92 \%$ of the total data, which is relatively low. If two-way ANOVA is further grouped, it will be difficult to draw credible conclusions due to insufficient data volume. Therefore, only oneway ANOVA is performed on the accuracy, as shown in Table 3.

Table 3. Analysis of the significance of each factor for the accuracy.

\begin{tabular}{c|c|c|c}
\hline \hline \multicolumn{2}{c|}{ Factor } & $\mathrm{F}$ & $\mathrm{P}$ \\
\hline \multicolumn{2}{c|}{ Orientation } & 0.527 & 0.746 \\
\hline \multirow{2}{*}{$\begin{array}{c}\text { Color } \\
\text { combination }\end{array}$} & Monochrome & 0.411 & 0.305 \\
\cline { 2 - 4 } & Two-tone & 33.257 & 0.004 \\
\hline \multicolumn{2}{c}{ Color grouping } & 16.349 & 0.001 \\
\hline \hline
\end{tabular}

The accuracy of the horizontal layout is $97.12 \%$, while the accuracy of the vertical layout is $96.57 \%$, which is $0.55 \%$ higher than the horizontal layout, which is in line with the speed accuracy principle. However, the impact of the orientation on the accuracy is not significant. In addition, the color combination has significant effect on accuracy $(f=16.349$, $\mathrm{p} \leq 0.001)$, in which the accuracy of monochrome form $(98.34 \%)$ is higher than the average accuracy of the two-color $(95.01 \%)$, which is $3.33 \%$ higher. The speed accuracy criteria is met, and the difference $(\mathrm{f}=8.326, \mathrm{p}=0.025)$ is significant.

Inter-group difference analysis is performed for each color groupings. As shown in Fig. 5 , the accuracy of the monochrome form is up to $98.34 \%$. For the two-color form, the accuracy of the $3 \times 4$ color grouping is $93.55 \%$, and the accuracy of the $6 \times 2$ is $98.05 \%, 1 \times 12$ has the second highest accuracy, which is up to $95.41 \%$. The differences between the groups are significant. As shown in Table 4 , only the $6 \times 2$ color grouping and the monochrome forms are not significantly different, and the monochrome and the $6 \times 2$ color grouping could be classified into one category. The styles of these two kinds of forms don't have much change in color and are highly similar, with a small error rate.

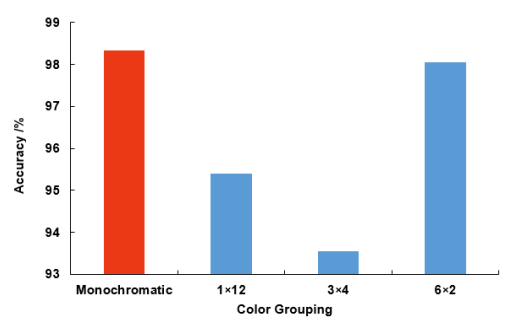

Fig. 5. Accuracy in different color groupings.

Table 4. Analysis of the difference between the accuracy in different color groupings.

\begin{tabular}{ccc}
\hline \hline \multirow{2}{*}{ Variance analysis combination } & \multicolumn{2}{c}{ variance analysis } \\
& $\mathrm{F}$ & $\mathrm{P}$ \\
\hline Monochrome \& $(1 \times 12)$ & 6.846 & 0.042 \\
Monochrome \& $(3 \times 4)$ & 25.225 & 0.001 \\
Monochrome \& $(6 \times 2)$ & 0.065 & 0.746 \\
$(1 \times 12) \&(3 \times 4)$ & 8.334 & 0.021 \\
$(1 \times 12) \&(6 \times 2)$ & 8.536 & 0.032 \\
$(3 \times 4) \&(6 \times 2)$ & 27.201 & 0.002 \\
\hline
\end{tabular}

For monochrome forms, the color attribute is not significantly different from the accuracy $(\mathrm{f}=0.912,0.254)$. As shown in Fig. 6 , the accuracy of blue is $98.34 \%$, the green is 
$97.86 \%$, and the red is $96.50 \%$. For the two-color form, unlike the monochrome, the color combination has a significant effect on the accuracy $(\mathrm{f}=25.332, \mathrm{p} \leq 0.01)$.

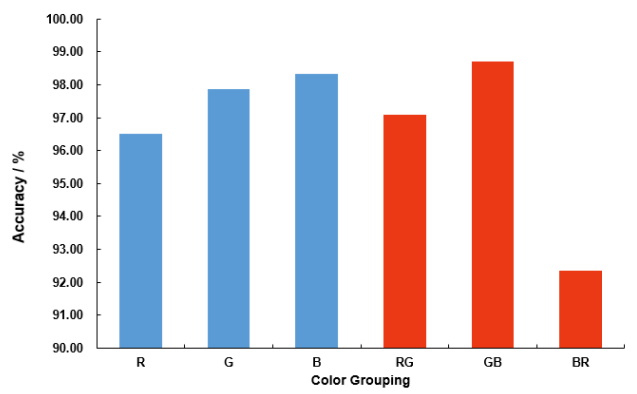

Fig. 6. Accuracy under each color attribute.

As shown in Table 5, the average accuracy of green-blue combination is up to $98.72 \%$, while the red-green combination is lower at $97.08 \%$, the blue-red combination is the least at $92.35 \%$ and significant difference exists between the other two color combinations. This means that the color combination has a stronger impact on the visual pre-attention process than a single color.

Table 5. Inter group difference analysis of accuracy at the different color combination.

\begin{tabular}{ccccc}
\hline \hline \multirow{2}{*}{ Color } & $\begin{array}{c}\text { Average correct } \\
\text { rate }\end{array}$ & Variance analysis & \multicolumn{2}{c}{ variance analysis } \\
& $97.08 \%$ & RG\&GB & 5.644 & 0.305 \\
RG & $98.72 \%$ & GB\&BR & 46.871 & 0.002 \\
GB & $92.35 \%$ & BR\&RG & 42.226 & 0.001 \\
BR & &
\end{tabular}

Table 6. A summary of the conclusions drawn from the experimental data.

\begin{tabular}{|c|c|c|c|}
\hline & Orientation & Color combination & Color grouping \\
\hline $\begin{array}{l}\text { Reaction } \\
\text { time }\end{array}$ & $\begin{array}{l}\text { - Orientation has a } \\
\text { significant impact on } \\
\text { the reaction time } \\
\text { - The horizontal } \\
\text { layout is faster than } \\
\text { the vertical layout }\end{array}$ & $\begin{array}{l}\text { - Color combination has } \\
\text { no significant impact on } \\
\text { the reaction time } \\
\text { - The average response } \\
\text { time of the two-color } \\
\text { form is less than the } \\
\text { monochrome Table }\end{array}$ & $\begin{array}{l}\text { - Color grouping has } \\
\text { a significant impact } \\
\text { on the reaction time } \\
\text { - Color grouping of } \\
6 \times 2 \text { has the best } \\
\text { performance on } \\
\text { reaction time }\end{array}$ \\
\hline Accuracy & $\begin{array}{lll}\text { - } & \text { The impact of the } \\
\text { orientation on the } & \text { on } \\
\text { accuracy is } & \text { not } \\
\text { significant } & & \\
\end{array}$ & 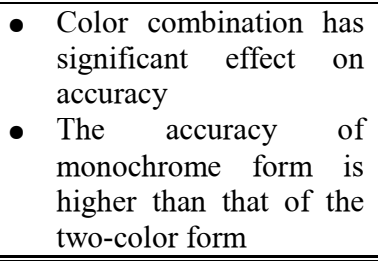 & $\begin{array}{l}\text { - Color grouping has } \\
\text { a significant impact } \\
\text { on the accuracy } \\
\text { - Color grouping of } \\
6 \times 2 \text { has the highest } \\
\text { accuracy }\end{array}$ \\
\hline
\end{tabular}

\section{Discussion}

The three factors (orientation, color grouping and color combination are used as the independent variables for analyzing the performance of interface. From the experimental results, the orientation has a significant impact on the reaction time, and the horizontal 
layout is $11.08 \%$ faster than the vertical layout, but the effect on the accuracy is not significant. The main reasons for the results are:

1) The visual system has a greater visual perception breadth than the vertical scan when performing the horizontal scan, and the lateral visual acuity declines more slowly.

Therefore, the horizontal visual search has a faster information processing speed [25] than the vertical.

2) People are more accustomed to horizontal scanning during visual scanning, and this increase in biological and habitual lateral scanning speed does not affect the accuracy of scanning. This is consistent with Gong Yong's result on the influence of the layout direction of the graphic panel on the recognition performance [26].

The color grouping has a significant impact on the recognition performance in reaction time and accuracy. The results show that the average reaction time of the monochrome form is $12.69 \%$ slower than the two-color form, and the accuracy is $3.33 \%$ higher. In the two-color form, the smaller the coloring density, the faster the reaction time and the difference between groups. The accuracy for $6 \times 2$ color grouping is the highest, while that of the $3 \times 4$ was the lowest, and the difference between the groups is significant. The main reasons for the results are:

1) For the information search of forms, the combination of the three colors of red, green and blue obviously has a stronger influence on people's pre-attention process than the use of one single color. Because the color difference brought by color combination is an independent feature that can be detected in the pre-attention stage, it helps to narrow the scope of visual search, and lay a foundation for the second processing process with memory limitations. While the monochrome form is too monotonous, the difference between the blocks in forms is not strong, and the recognition efficiency is not high, so the reaction time is long.

2) Grouping strategy for information processing. As mentioned in the Section 2, color is the most important factor affecting the pre-attention phase. The subjects prioritized the processing stage to group the information in forms according to the color, and then systematically searched the information in the subsequent sequence processing stage, and the memory capacity of the person was limited during the sequence processing stage. The span of human short-term memory can only cover $7 \pm 2$ blocks of information [27]. This principle is applied to interaction design, and document filling to improve navigation and search efficiency. In this experiment, the form of $6 \times 2$ color grouping is divided into two groups, each of which has 6 blocks that are made up of numbers and Chinese characters. These 6 blocks are just well matched with the span of human short-term memory, so they are processed together in parallel. The line of sight does not oscillate back and forth because of the color classification, so the reaction time is the shortest and the accuracy is highest in the two-color form. The $3 \times 4$ color grouping is divided into 4 blocks, each of which contains 3 components. The subjects also perform visual search based on the color grouping strategy, and then there are three other color components between the color blocks. Working memory causes interference. The visual search method has a back-and-forth swing phenomenon, so the reaction time is longer than the $6 \times 2$ color grouping, and the accuracy is low. For the $1 \times 12$, since its color conversion is too frequent, its grouping property is not obvious, and there are two kinds of visual search methods. One is a grouping-based search method, and the other is a full-sequence search method that ignores grouping policies. The search method based on the grouping strategy will have a short response time, and due to the existence of the grouping interval, the accuracy will also be reduced. The full sequence search method has a long reaction time but the highest accuracy. Therefore, in general, the reaction time of $1 \times 12$ is only faster than the monochrome, and the accuracy is higher than the $3 \times 4$ color grouping. 
In general, whether it is a single color or a combined color has no significant effect on the reaction time, and the combined color has a significant influence on the accuracy. Among them, the accuracy of green and blue combination is $98.72 \%$, and the accuracy of blue-red combination color is only $92.35 \%$. Christ [28] summarized 42 literatures on color impact display interface search performance between 1952 and 1973 and found that color usage has a positive impact on search performance in certain circumstances and negative effects in some environments. This indicates that the environment is an important factor influencing the visual impact of color.

To summarize the experimental procedure of this paper, on the one hand, the combination of the two of four Chinese characters "You", "Shen", "Jia" and "Tian" is difficult to identify and it is not an objective of this study to compare the results based on the level of difficulty, but it has created a bottom line effect for the influence of color in the visual search. So the color has little effect on the reaction time [29]. On the other hand, due to the combined use of colors, the degree of discrimination between chunks is improved, and the influence on the visual search process is more intense. When searching for a twocolor form, the subject may abandon the accuracy in order to ensure the reaction time, so the significant effect of the combined color is reflected in the accuracy. In addition, the color grouping, color combination, and orientation are not significant for the interaction of the reaction time, so no further discussion is made.

\section{Conclusion}

Through the analysis above, the following conclusions can be drawn:

1) When designing the interfaces of different systems that have a lot of forms, such as weapon command and control system, nuclear power plant monitoring system, you can use the horizontal layout as much as possible, and you can color the forms with multiple colors. The coloring should take into account of the short-term memory characteristics of humans. A coloring block can contain $7 \pm 2$ components, which can speed up the reaction while ensuring a higher accuracy.

2) The design should be combined with the use of the environment to fully consider the effects of color and color combination. It is known in the text that in the design of forms, for the color combination, the green-blue combination is superior to the red-green combination and the red-blue combination.

In this study we only use three colors of red, green and blue which are applied in the display interface. Combining them in two, only three combinations of colors, the analysis of the combined colors in the form is not enough, and the combination of colors is one of the most important factors affecting visual search performance [30]. Furthermore, in the display interface, various types of charts are displayed intricately, and the display of the Table and other display elements should be considered to ensure the coordination of the entire display interface. In addition, this study is mainly based on the analysis of behavioral performance, and does not have subjective and objective evaluation of the form design. In the later research, on the one hand, it is necessary to fully consider the influence of more color combinations and display elements. On the other hand, it is necessary to combine the subjective evaluation, eye movement measurement and other research methods to further explore the design guidelines for visual search charts to accord with cognitive characteristics, reduce cognitive load, and improve interaction efficiency. Furthermore, it provides guidelines for the ergonomic design of the display interface of submarine, ship, anti-aircraft missile and other types of display control systems. 


\section{Reference}

1. L. Gutterman, "Next Generation Armament Test Solutions for the Flightline," 2018 IEEE AUTOTESTCON, National Harbor, MD, 2018, pp. 1-4.

2. L. Leilei, W. Hongxin, X. Yubing, Y. Zhenshan, Y. Chenyi and Y. Fan, "Research and development for landing gear test interface unit for one type aircraft," CSAA/IET International Conference on Aircraft Utility Systems (AUS 2018), Guiyang, 2018, pp. 216-219.

3. Q. Zeng, B. Jiang and Q. Duan, "Integrated evaluation of hardware and software interfaces for automotive human-machine interaction," in IET Cyber-Physical Systems: Theory \& Applications, vol. 4, no. 3, pp. 214-220, Sep. 2019.

4. Y.X. Zhang, K. Z. Yang, M.C. Song, and M. Yang, "Human error analysis of starting CVCS in soft controls in nuclear power plants," J. Harbin. Eng. Univ., vol. 37, no. 12, pp. 1653-1657, Nov. 2016.

5. S.Y. Yan, Human factors in weapons and equipment. Harbin, China: Harbin Inst.Techno Press, 2009, pp. 16-20.

6. K. L. Mosier, “Automation, task, and context features: Impacts on pilots' judgments of human-automation interaction,” J. Cogn. Eng. Decis. Mak., vol. 7, no. 4, pp. 377-399, 2013.

7. K. B. Sulliven, "Using neural networks to assess flight deck human-automation interaction,” Reliab. Eng. Syst. Safe, vol. 114, no. 1, pp. 26-35, Jun. 2013.

8. W. Y. Kang et al, "Optimization design of vision display interface in plane cockpit based on mental workload," J. B. Univ. Aeronaut. Astronaut., vol. 34, no. 7, pp.782785, Jul. 2008.

9. X. L. Fan, Q. X. Zhou, Z. Q. Liu, "Principle of plane display interface design based on visual search,” J. B. Univ. Aeronaut. Astronaut., vol. 41, no. 2, pp. 216-221, Jul. 2014.

10. Z. M. Wei, X. R. Wanyan, D. M. Zhuang, "Measurement and evaluation of mental workload for aircraft cockpit display interface," J. B. Univ. Aeronaut. Astronaut., vol. 40, no. 1, pp. 86-91, May. 2015.

11. H. S. Zhang, D. M. Zhuang, "The Study on pleasure and ergonomics of cockpit interface design," in Proc. CAID\&CD2009, Wenzhou, China, 2009, pp. 1400-1402.

12. A. Murata, N. Furukawa, "Relationships among display features, eye movement characteristics, and reaction time in visual search," Hum. Factors, vol. 47, no. 3, pp. 598-612, Sept. 2005.

13. T. Lindberg, R. Nasanen, "The effect of icon spacing and size on the speed of icon processing in the human visual system," Displays, vol. 24, no. 3, pp. 111-120, Oct. 2003.

14. P. V. Schaik, J. Ling, "Design parameters in web pages: frame location and differential background contrast in visual search performance," Int. J. Ind. Ergonom., vol. 5, no. 3, pp. 459-471, 2001.

15. A. Chalbi et al., "Common Fate for Animated Transitions in Visualization," in IEEE T. VIS. COMPUT. GR., vol. 26, no. 1, pp. 386-396, Jan. 2020.

16. R. Nasanen, R. Ojanpaa, I. Kojo, "Effect of stimulus contrast on performance and eye movements in visual search," Vision Res., vol. 41, no. 14, pp. 1817-1824, Jun. 2001.

17. M. Niemela, J. Saarinen. "Visual search for grouped versus ungrouped icons in a computer interface," Hum. Factors, vol. 42, no. 4, pp. 630-635, Dec. 2000. 
18. Y. Q. Liang, P. L. Li, W. Wang, "Design Method of Matching Icon Utilization and Easy Search Rate in GUI," J. Comput.-Aided Design \& Comput. Graphics, vol. 30, no. 1, pp. 155-162, Feb. 2018.

19. J. M. Wolfe, "Forty years after feature integration theory: An introduction to the special issue in honor of the contributions of Anne Treisman," Attention. Percept. Psycho., vol. 82, no. 1, pp. 1-6, 2020.

20. N. P. Bichot, S. C. Rao, J. D. Schall, "Continuous processing in macaque frontal cortex during visual search," Neuropsychologia, vol. 39, no. 9, 2001.

21. J. M. Wolfe, T. S. Horowitz, "What attributes guide the deployment of visual attention and how do they do it? ," Nat. Rev. Neurosci., vol. 5, no. 6, pp. 495-501, Jun. 2004.

22. H. Y. Wang et al, "Analysis of Cognitive Model in Icon Search Behavior Based on ACT-R Model,” J. Comput.-Aided Design \& Comput. Graphics, vol. 28, no. 10, pp. 1740-1749, Oct. 2016.

23. Y. Q. Liang, P. L. Li, W. Wang, "Design Method of Matching Icon Utilization and Easy Search Rate in GUI," J. Comput.-Aided Design \& Comput. Graphics, vol. 30, no. 1, pp. 155-162, Feb. 2018.

24. Y. T. Jin, J Lv, W, J. Pan, "Layout optimization of virtual interactive interface based on visual attention mechanism," Comput. Eng. Desig., vol. 41, no. 03, pp. 763-769, Mar. 2020.

25. M. H. Pillips, J. A. Edelman, "The dependence of visual scanning performance on search direction and difficulty," Vision Res., vol. 48, no. 21, pp. 2184-2192, Sep. 2008.

26. Y. Gong et al., "Effects of graphical panel layout characteristics on human-computer interactive efficiency," J. Comput.-Aided Design \& Comput. Graphics, vol. 24, no. 9, pp. 1145-1150, Sep. 2012.

27. Y. Zhang, N. J. Liang, "A study on the short-term memory of numerals in the different background noise,” Psychol. Sci., no. 4, pp. 789-794, 2006.

28. R. E. Christ. "Review and analysis of color coding research for visual displays," Hum. Factors, vol. 17, no. 6, pp. 542-570, Dec. 1975.

29. D. Zheng, H. Wang, J. Wang, X. Zhang and W. Chen, "Toward Visibility Guaranteed Visual Servoing Control of Quadrotor UAVs," in IEEE/ASME Transactions on Mechatronics, vol. 24, no. 3, pp. 1087-1095, June 2019.

30. Y. Gong et al. "Effect of Color Combination on Graphical Symbol Visual Search Efficiency," J. Comput.-Aided Design \& Comput. Graphics, vol. 28, no. 7, pp. 11151120, Jul. 2016. 